\title{
Mini Commentary
}

\section{6-OG-17850R1 Women's autonomy versus societal pressure to control fertility}

\section{S Rowlands}

Centre of Postgraduate Medical Research and Education, Bournemouth University, Bournemouth, UK

An important study by Aiken and colleagues published in this issue of BJOG examines women's preferences for post-TOP contraception, not just the method they do in fact receive. A striking finding is that, when all women undergoing TOP are offered impartial information on the full range of contraceptives, only one half actively seek supply of their chosen method from their TOP provider. One third of women undergoing TOP wish to obtain their method of choice from another provider and the rest state that they do not need contraception or do not wish to discuss contraception. As the authors state, women's choices to consult with their preferred clinician about contraception should be respected. Public health specialists should take note that the denominator for measurement of contraception provision at TOP is only about one half of the women who proceed with a TOP. It seems that expectations of what TOP providers can achieve in terms of delivery of contraception are often unrealistically high.

Bearing in mind rapid return to fertility following TOP and poor attendance rates when follow-up is offered, same-day delivery of contraception is an ideal model. An exception to this is intrauterine contraception (IUC) for the increasing proportion of women having a medical, as opposed to a surgical, TOP. Many services defer IUC insertion for 1 - 4 weeks after medical TOP, although evidence suggests that it can be done as soon as the products of conception have been passed (GemzellDanielsson et al. Int J Gyn Obstet 2014; 126: S52-55). However, because with medical TOP before nine weeks' gestation passage of products usually takes place outside a medical facility, insertion has to be done at follow-up. With the increasing popularity of telephone follow-up after medical TOP this would involve an extra attendance. This means that take-up of IUC is bound to be lower after medical TOP compared with surgical TOP when insertion can be done intra-operatively; the Aiken 
study confirms this. More research is needed on the optimal timing of initiation of IUC after medical TOP.

The authors also point out that the extent to which method-specific contraceptive uptake targets reflect women's preferences has not been researched. There is a fine balance between promotion of highly-effective contraception to reduce unintended pregnancies, in particular more than one such pregnancy, as a public health measure and regard for individual dignity. Women's autonomy should be respected through free, full and informed decision-making (Ensuring human rights in the provision of contraceptive information and services Geneva: WHO; 2014). Clinicians need to be mindful that, once women requesting TOP have been informed about their fertility and given options and opportunities to control it, their right to self-determination overrides any policies and targets currently being handed down.

\section{Disclosure of interests}

The author has no conflicts of interest to report. 